\title{
Invariant Solutions of Two Dimensional Heat Equation
}

\author{
Narmanov Otabek Abdigapparovich \\ Department of Algorithms and Mathematical Modelling, Tashkent University of Information Technologies, Tashkent, Uzbekistan \\ Email: otabek.narmanov@mail.ru
}

How to cite this paper: Abdigapparovich, N.O. (2019) Invariant Solutions of Two Dimensional Heat Equation. Journal of Applied Mathematics and Physics, 7, 1488-1497. https://doi.org/10.4236/jamp.2019.77100

Received: May 14, 2019

Accepted: July 16, 2019

Published: July 19, 2019

Copyright $\odot 2019$ by author(s) and Scientific Research Publishing Inc. This work is licensed under the Creative Commons Attribution International License (CC BY 4.0).

http://creativecommons.org/licenses/by/4.0/

\section{c) (i) Open Access}

\begin{abstract}
The symmetry group of a given differential equation is the group of transformations that translate the solutions of the equation into solutions. If the infinitesimal generators of symmetry groups of systems of partial differential equations are known, the symmetry group can be used to explicitly find particular types of solutions that are invariant with respect to the symmetry group of the system. The class of invariant solutions includes exact solutions that have direct mathematical or physical meaning. In this paper, using the well-known infinitesimal generators of some symmetry groups of the two-dimensional heat conduction equation, solutions are found that are invariant with respect to these groups. It is considered cases when conductivity coefficients of the two-dimensional heat conduction equation are power functions of temperature and conductivity coefficients are exponential functions of temperature. In first case invariant solutions contain well known self-similar solutions which are widely used in applications.
\end{abstract}

\section{Keywords}

Symmetry Group, Heat Equation, Infinitezimal Generator, Vector Field

\section{Introduction}

Let us be given a differential equation of order $m$

$$
\Delta\left(x, u^{(m)}\right)=0
$$

from $n$ independent $x=\left(x^{1}, x^{2}, \cdots, x^{n}\right)$ and $q$ dependent $u=\left(u^{1}, u^{2}, \cdots, u^{q}\right)$ variables containing derivatives from $u$ with $x$ to order $m$.

Definition. A group $G$ of transformations acting on the set $M$ of the space of independent and dependent variables of a differential equation is called the symmetry group of Equation (1) if for each solution $u=f(x)$ of Equation (1) 
and for $g \in G$ such that $g \circ f$, is defined, then the function $\tilde{u}=g \circ f$, is also a solution of the Equation (1).

For the heat equation $u_{t}=u_{x x}$ translation group

$$
(x, t, u) \rightarrow(x+a s, t+b s, u), s \in \mathbb{R}
$$

is a symmetry group, because if the function $u=f(x)$ is a solution, then the function $u=f(x-a s, t-b s)$ is also a solution to the heat equation.

One of the advantages of knowing the symmetry group of differential equations is that if we know the solution $u=f(x)$, then according to the definition of symmetry group the function $\tilde{u}=g \circ f$ is also a solution for any element $g$ groups $G$, so that we have the opportunity to build a whole family of solutions, exposing the known solution to the action of all possible elements of the group.

Group analysis methods are widely used for the study of partial differential equations and for the integration of ordinary differential equations. The papers [1] [2] [3] [4] deal with the integration of ordinary differential equations and linear differential equations in partial derivatives, based on known infinitesimal symmetries.

Numerous studies have been devoted to finding symmetry groups of differential equations and their applications for research. The work [5] has developed a computational method that explicitly defines the full symmetry group of an arbitrary partial differential equation. In the paper [6] there are considered problems of group classification of differential equations and their solutions. Examples of the application of group analysis techniques to specific systems of differential equations are given. In the paper [7] it was found the Lie algebra of infinitesimal generators of the symmetry group for the two-dimensional and three-dimensional heat equation. The Lie algebra of infinitesimal generators of the symmetry group for the one-dimensional heat equation was used in [8].

Using of infinitely generators of some symmetry groups allows us to consider ordinary differential equations instead of partial differential equations. If we choose suitable invariant functions, in a fairly large class of cases, these ordinary differential equations are integrated into quadratures or solutions of differential equations are expressed by well-known functions. The solutions obtained include exact solutions that have direct mathematical or physical meaning.

We present methods for obtaining invariant solutions that describe the process of heat distribution fairly well using well-known infinitesimal generators of symmetry groups. Some invariant solutions of the two-dimensional heat conduction equation are found in the paper [7] when conductivity coefficients are with power functions with a negative exponent. We consider the case when conductivity coefficients are power functions with a positive exponent and exponential functions of temperature.

\section{Conductivity Coefficients Are Power Functions of Temperature}

Let us consider the two-dimensional heat equation 


$$
u_{t}=\sum_{i=1}^{2} \frac{\partial}{\partial x_{i}}\left(k_{i}(u) \frac{\partial u}{\partial x_{i}}\right)+Q(u)
$$

where $u=u\left(x_{1}, x_{2}, t\right) \geq 0$-is the temperature function, $k_{i}(u) \geq 0, Q(u)$ -functions of temperature $u$. The function $Q(u)$ describes the process of heat generation if $Q(u)>0$ and the process of heat absorption if $Q(u)<0$.

Studies show that the thermal conductivity coefficients $k_{1}(u), k_{2}(u)$ in a fairly wide range of parameters can be described by a power function of temperature, i.e. it has the form $k(u)=u^{\sigma}$ [2] [9].

Consider the case $k_{1}(u)=k_{2}(u)=u^{\sigma}, Q(u)=u$. In this case, Equation (3) has the following form:

$$
u_{t}=u^{\sigma} \Delta u+\sigma u^{\sigma-1}(\nabla u)^{2}+u
$$

where $\Delta u=\frac{\partial^{2} u}{\partial x_{1}^{2}}+\frac{\partial^{2} u}{\partial x_{2}^{2}}$-Laplace operator, $\nabla u=\left\{\frac{\partial u}{\partial x_{1}}, \frac{\partial u}{\partial x_{2}}\right\}$ - the gradient of the function $u$.

As shown in [7], the following vector fields are infinitesimal generators of the symmetry group for Equation (4):

$$
X_{1}=\sigma x_{1} \frac{\partial}{\partial x_{1}}+\sigma x_{2} \frac{\partial}{\partial x_{2}}+2 u \frac{\partial}{\partial u}, X_{2}=\exp (-\sigma t) \frac{\partial}{\partial t}+\exp (-\sigma t) u \frac{\partial}{\partial u} .
$$

The flows of the vector fields $X_{1}, X_{2}$ generate the following transformation groups, respectively

$$
\begin{gathered}
\left(t, x_{1}, x_{2}, u\right) \rightarrow\left(t, x_{1} \mathrm{e}^{s}, x_{2} \mathrm{e}^{s}, u \mathrm{e}^{2 s}\right), s \in \mathbb{R} \\
\left(t, x_{1}, x_{2}, u\right) \rightarrow\left(\frac{1}{\sigma} \ln \left(\mathrm{e}^{\sigma t}+\sigma s\right), x_{1}, x_{2}, u\left(\mathrm{e}^{\sigma t}+\sigma s\right)^{\frac{1}{\sigma}}\right), s \in \mathbb{R}
\end{gathered}
$$

We find solutions of Equation (4) that are invariant with respect to transformation groups (6), (7). To do this, we first find the invariant functions of these transformations.

It is known that [[5], p. 117] a smooth function $f: M \rightarrow R$ is an invariant function of the transformation group $G$, acting on $M$ if and only if $X f=0$ for each infinitesimal generator $X$ of the group $G$.

Using this criterion, we find that the functions

$$
I_{1}=\frac{\left(x_{1}+x_{2}\right) \exp (\sigma t / 2)}{\sqrt{u^{\sigma}}}, I_{2}=\frac{x_{1}}{x_{2}}
$$

are invariant functions of the transformation group (6), (7), which follows from the following equalities $X_{1}\left(I_{1}\right)=0, X_{1}\left(I_{2}\right)=0, X_{2}\left(I_{1}\right)=0, X_{2}\left(I_{2}\right)=0$.

These invariant functions allow us to search solution of Equation (4) in the form

$$
u\left(t, x_{1}, x_{2}\right)=\frac{\sigma}{2} \mathrm{e}^{t} \frac{\left(x_{1}+x_{2}\right)^{2 / \sigma}}{2} V(\xi)
$$

where $\xi=\frac{x_{1}}{x_{2}}$. Substituting in (4) for the function $V(\xi)$ we obtain the fol- 
lowing second-order differential equation:

$$
\begin{aligned}
& f(\xi) V V^{\prime \prime}+f(\xi) V^{\prime 2}+4 \sigma(\xi+1)\left[\frac{\sigma}{2}\left(\xi^{2}+\xi\right)-2 \xi+2\right] V V^{\prime} \\
& +4\left[2+2\left(\frac{2}{\sigma}-1\right)\right] V^{2}=0
\end{aligned}
$$

where $f(\xi)=(\xi+1)^{2}\left(\xi^{2}+1\right), g(\xi)=\sigma(\xi+1)\left[\frac{\sigma}{2}\left(\xi^{2}+\xi\right)-2 \xi+2\right]$.

In the general case for any $\sigma$ Equation (10) by replacing $z(\xi)=V^{2}$ can be reduced to a second-order linear equation for $z(\xi)$.

In the case of $\sigma=2$, we obtain the following equation

$$
\begin{gathered}
f(\xi) V V^{\prime \prime}+f(\xi) V^{\prime 2}+2 g(\xi) V V^{\prime}+8 V^{2}=0, \\
g(\xi)=4(\xi+1)\left(\xi^{2}-\xi+2\right) .
\end{gathered}
$$

In Equation (11) introducing a new function $z(\xi)$ by means of the equality $z(\xi)=V^{2}$ we obtain the following second order linear equation for $z(\xi)$

$$
f(\xi) \frac{\mathrm{d}^{2} z}{\mathrm{~d} \xi^{2}}+4 g(\xi) \frac{\mathrm{d} z}{\mathrm{~d} \xi}+16 z=0
$$

In this equation making the substitution $w(x)=z \exp \left[2 \int \frac{g}{f} \mathrm{~d} \xi\right]$ get the equation

$$
w^{\prime \prime}+I(\xi) w=0 .
$$

Then, making the replacement $p(\xi)=\frac{w^{\prime}}{w}$, we obtain the equation

$$
p^{\prime}+p^{2}=-I(\xi)
$$

where $I=-\frac{6\left(\xi^{2}-2 \xi+1\right)}{(\xi+1)^{2}\left(\xi^{2}+1\right)}$.

This equation is the Riccati equation. It is known that if we know one particular solution of the Riccati equation, we can find a general solution this equation.

Now consider the case when there is heat absorption:

$k_{1}(u)=k_{2}(u)=u^{\sigma}, Q(u)=-u$. In this case, the Equation (3) has the following form:

$$
u_{t}=u^{\sigma} \Delta u+\sigma u^{\sigma-1}(\nabla u)^{2}-u
$$

As shown in [7], the following vector fields are infinitesimal generators of the symmetry group for Equation (15):

$$
X_{1}=\sigma x_{1} \frac{\partial}{\partial x_{1}}+\sigma x_{2} \frac{\partial}{\partial x_{2}}+2 u \frac{\partial}{\partial u}, X_{2}=\exp (\sigma t) \frac{\partial}{\partial t}+\exp (\sigma t) u \frac{\partial}{\partial u} .
$$

Using the above criterion, we find that the functions

$$
I_{1}=\frac{\left(x_{1}+x_{2}\right) \exp (-\sigma t / 2)}{\sqrt{u^{\sigma}}}, I_{2}=\frac{x_{1}}{x_{2}}
$$


are invariant functions of the transformation groups generated by vector fields (16), which follows from the following equalities $X_{1}\left(I_{1}\right)=0, X_{1}\left(I_{2}\right)=0$, $X_{2}\left(I_{1}\right)=0, X_{2}\left(I_{2}\right)=0$.

These invariants allow us to search a solution of Equation (15) in the form

$$
u\left(t, x_{1}, x_{2}\right)=\frac{\sigma}{2} \mathrm{e}^{-t} \frac{\left(x_{1}+x_{2}\right)^{2 / \sigma}}{2} V(\xi)
$$

Substituting function (18) in (15) for the function $V(\xi)$, we obtain the second-order differential equation, which coincides with Equation (10).

Thus, in both cases, to find solutions that are invariant with respect to transformation groups, we must investigate a second-order linear equation with respect to $V(\xi)$, which depends on $\sigma$. As shown by numerical studies, the solution of Equation (10) is limited (see Figure 1). Therefore, at each point of the domain of variables $\left(x_{1}, x_{2}\right)$, different from points $(0,0)$, temperature function (9) increases exponentially with increasing $t$, that characterizes the presence of a heat source in Equation (4). In Equation (15) there is an absorption source, therefore at every point of the domain of variables $\left(x_{1}, x_{2}\right)$, other than $(0,0)$, the temperature function (18) decreases exponentially as $t$ increases.

Now consider the case when there is no heat source: $k_{1}(u)=k_{2}(u)=u^{\sigma}$, $Q(u)=0$. In this case, Equation (3) has the following form:

$$
u_{t}=u^{\sigma} \Delta u+\sigma u^{\sigma-1}(\nabla u)^{2}
$$

Let us show one family of invariant solutions of Equation (19), which is often used in applications. For this we use the infinitesimal symmetry of Equation (19), which is given by the following vector field

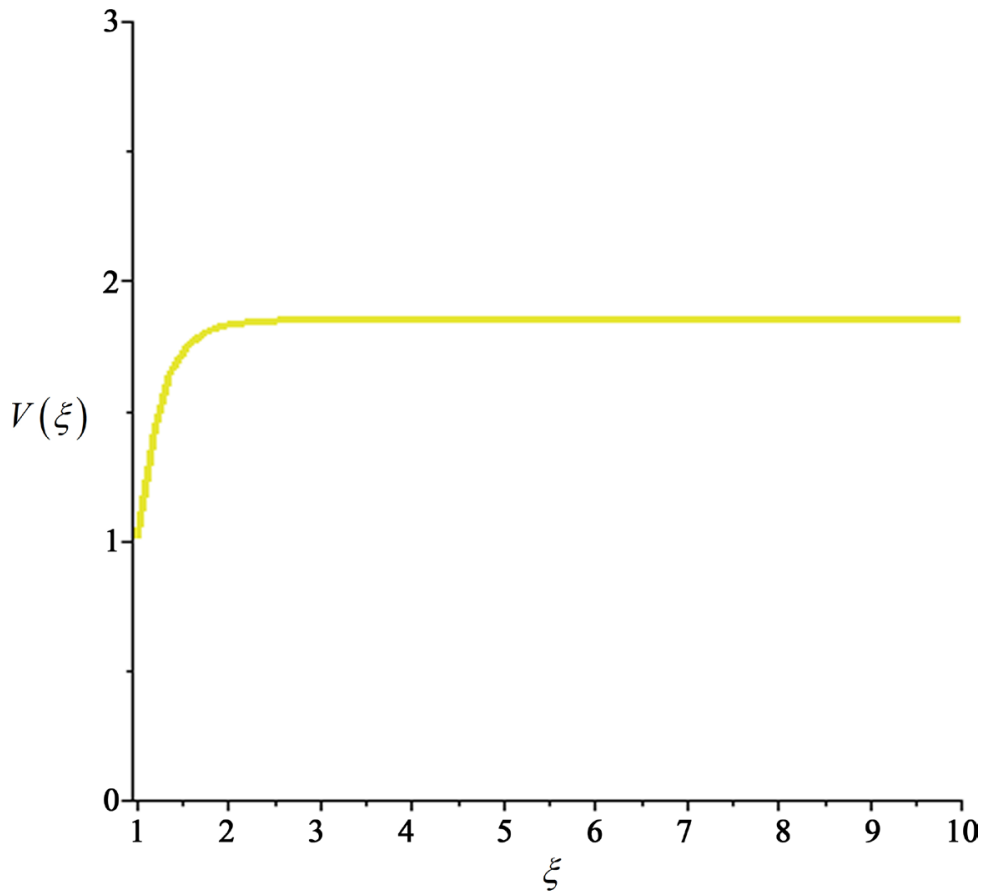

Figure 1. Grafics of the solution of Equation (10) (function $V(\xi))$ at $V(1)=1, V^{\prime}(1)=10$ ). 


$$
X_{1}=-x_{2} \frac{\partial}{\partial x_{1}}+x_{1} \frac{\partial}{\partial x_{2}} \text {. }
$$

The flow of this vector field consists of rotations around the origin of the plane $\left(x_{1}, x_{2}\right)$, which define the following one parameter transformation group

$$
\left(t, x_{1}, x_{2}, u\right) \rightarrow\left(t, x_{1} \cos s-x_{2} \sin s, x_{1} \sin s+x_{2} \cos s, u\right), s \in \mathbb{R} \text {. }
$$

Invariant functions of these rotations are functions.

$$
I_{1}=u f(f), I_{2}=g(t)\left(x_{1}^{2}+x_{1}^{2}\right)^{1 / 2},
$$

where $f(t), g(t)$ are smooth functions.

Using these functions, we can find a large family of invariant solutions of Equation (19), relative to the groups of rotations around the origin of the plane $\left(x_{1}, x_{2}\right)$.

We are looking for a solution in the form

$$
u\left(t, x_{1}, x_{2}\right)=t^{\alpha} V(\xi), \xi=t^{-\beta}\left(x_{1}^{2}+x_{1}^{2}\right)^{1 / 2},
$$

to get self-similar solutions [9].

Substituting (23) into Equation (19), we obtain the following relation $\alpha-1=\alpha(\sigma+1)-2 \beta$. For solutions of this type usually require the condition of constancy of energy, that is, the condition:

$$
\iint_{R^{2}} u\left(t, x_{1}, x_{2}\right) \mathrm{d} x_{1} \mathrm{~d} x_{2}=\text { const }=E .
$$

From condition (24) we obtain the equality $\alpha+2 \beta=0$. Thus, we obtain $\alpha=-2 /(2 \sigma+2), \quad \beta=1 /(2 \sigma+2)$.

As a result, we obtain the following differential equation for the function $V(\xi)$ :

$$
\xi V^{\sigma} V^{\prime \prime}+\xi \sigma V^{\sigma-1} V^{\prime 2}+V^{o} V^{\prime}+\frac{\xi^{2}}{2 \sigma+2} V^{\prime}+\xi \frac{1}{\sigma+1} V=0
$$

This equation is equivalent to the following equation.

$$
\left(\xi V^{o} V^{\prime}\right)^{\prime}+\frac{1}{2 \sigma+2}\left(V \xi^{2}\right)^{\prime}=0 .
$$

By declining $V \neq 0$ and considering the integration constant to be zero, from Equation (26) we get the following expression for $V$

$$
V(\xi)=\left[\frac{\sigma}{4(\sigma+1)}\left(C^{2}-\xi^{2}\right)\right]^{\frac{1}{\sigma}} .
$$

Supplying the expression for $V$ in (23) we obtain the following solution, which is invariant with respect to rotations around the origin of the plane $\left(x_{1}, x_{2}\right)$ :

$$
u\left(t, x_{1}, x_{2}\right)=\frac{1}{\frac{1}{t^{\sigma+1}}}\left[\frac{\sigma}{4(\sigma+1)}\left(C^{2}-\frac{x_{1}^{2}+x_{2}^{2}}{\frac{1}{t^{\sigma+1}}}\right)\right]^{\frac{1}{\sigma}} \text {. }
$$

If we take into account that the $u\left(t, x_{1}, x_{2}\right) \geq 0$, function $u\left(t, x_{1}, x_{2}\right)$ is finite 
in $x=\left(x_{1}, x_{2}\right)$ and infinitely differentiable in the domain $\frac{x_{1}^{2}+x_{2}^{2}}{t^{\frac{1}{\sigma+1}}}<C^{2}$. Therefore, the integral in (24) is finite.

If we introduce the variables $\xi^{1}=\frac{x_{1}}{t^{\beta}}, \xi_{2}=\frac{x_{2}}{t^{\beta}}$, then equality (24) takes the form:

$$
\iint_{0 \leq \xi_{1}^{2}+\xi_{2}^{2} \leq C^{2}} V(\xi) \mathrm{d} \xi_{1} \mathrm{~d} \xi_{2}=\text { const }=E .
$$

where do we get that

$$
C=\left[\frac{1}{\pi}\left[4 \frac{\sigma+1}{\sigma}\right]^{\frac{1}{\sigma}}\left(\frac{1}{\sigma}+1\right) E\right]^{\sigma /(2 \sigma+2)} .
$$

In Equation (19) there is no source of absorption and no source of heat release, therefore, at each point in the domain of variables $\left(x_{1}, x_{2}\right)$ the temperature function (28) decreases uniformly with increasing $t$.

\section{Conductivity Coefficients Are Exponential Functions of Temperature}

Now consider the case when the thermal conductivity coefficients $k_{1}(u), k_{2}(u)$ are described by an exponential function of temperature, that is, they have the forms $k_{1}(u)=k_{2}(u)=\exp (u)$.

First consider the case when there is no heat source: $k_{1}(u)=k_{2}(u)=\exp (u)$, $Q(u)=0$. In this case, Equation (3) has following view:

$$
u_{t}=\exp (u) \Delta u+\exp (u)(\nabla u)^{2} .
$$

where $\Delta u=\frac{\partial^{2} u}{\partial x_{1}^{2}}+\frac{\partial^{2} u}{\partial x_{2}^{2}}$-Laplace operator, $\nabla u=\left\{\frac{\partial u}{\partial x_{1}}, \frac{\partial u}{\partial x_{2}}\right\}$ - the gradient of the function $u$.

As shown in [7], the following vector field is infinitesimal generators of the symmetry group for Equation (31):

$$
X=t \frac{\partial}{\partial t}-\frac{\partial}{\partial u},
$$

Theorem 1. The invariant solutions of Equation (31), with respect to the group of transformations, generated by the vector field (32) are functions

$$
u\left(t, x_{1}, x_{2}\right)=\ln \frac{V(\xi)}{t}
$$

where $V(\xi)=C_{1} \ln \xi-\xi+C_{2}, \quad \xi=\frac{x_{1}^{2}+x_{2}^{2}}{4}, C_{1}, C_{2}$-are arbitrary constants.

Proof. The flow of the vector field $X$ (32) generates the following transformation group of the space of variables $\left(t, x_{1}, x_{2}, u\right)$, for which the solutions of Equation (31) are invariant:

$$
\left(t, x_{1}, x_{2}, u\right) \rightarrow\left(t \mathrm{e}^{s}, x_{1}, x_{2}, u-s\right), s \in \mathbb{R}
$$


We find solutions of Equation (31) that are invariant with respect to transformation groups (34).

To do this, we first find the invariant functions of these transformations using above criterion.

The function $I_{1}=\frac{\exp (u / 2) \sqrt{t}}{V(\xi)}, \xi=\xi\left(x_{1}, x_{2}\right)$, is an invariant function of the group of transformations generated by the flow of the vector field (32), which follows from the following equality $X_{1}\left(I_{1}\right)=0$.

The solution of Equation (31) is sought in the form

$$
u\left(t, x_{1}, x_{2}\right)=\ln \frac{V(\xi)}{t}
$$

$\xi=\frac{x_{1}^{2}+x_{2}^{2}}{4}$. Then for the function $V(\xi)$ we get the following second-order differential equation

$$
\xi V^{\prime \prime}+V^{\prime}+1=0
$$

where do we find that $V(\xi)=C_{1} \ln \xi-\xi+C_{2}, \quad \xi=\frac{x_{1}^{2}+x_{2}^{2}}{4}$.

Now consider the case when there is a source of heat. Let $k_{1}(u)=k_{2}(u)=\exp (u), Q(u)=\exp (u)$. In this case, Equation (3) has the following form:

$$
u_{t}=\exp (u) \Delta u+\exp (u)(\nabla u)^{2}+\exp (u)
$$

As shown in [7], the following vector field is the infinitesimal generator of the symmetry group for Equation (37):

$$
X=2 t \frac{\partial}{\partial t}-2 \frac{\partial}{\partial u}
$$

This means that the flows of these vector fields generate a group of transformations of the space of variables $\left(t, x_{1}, x_{2}, u\right)$, which translate the solutions of Equation (37) into solutions.

Let us recall solution of a differential equation of the form

$$
x^{2} y^{\prime \prime}(x)+x y^{\prime}(x)+\left(x^{2}-v^{2}\right) y(x)=0
$$

is called the Bessel function of the index $v$. A large number of a wide variety of tasks related practically all the most important branches of mathematical physics and those designed to answer topical technical questions are connected with the use of Bessel functions. Bessel functions are widely used in solving problems of acoustics, radiophysics, hydrodynamics, problems of atomic and nuclear physics. There are numerous applications of Bessel functions to the theory of heat conduction. Such popularity of Bessel functions is explained by the fact that equations of mathematical physics containing Laplace operator in cylindrical coordinates, by the classical method separation of variables lead to the ordinary differential potential equation to determine these functions.

Following theorem shows that solutions of the Equation (37) can de described 
by Bessel functions.

Theorem 2. Functions

$$
u\left(t, x_{1}, x_{2}\right)=\ln \frac{V(\xi)}{t}
$$

where $V(\xi)=C_{1} \operatorname{BesselJ}(0,2 \sqrt{\xi})+C_{2} \operatorname{Bessel} Y(0,2 \sqrt{\xi})+1, \quad \xi=\frac{x_{1}^{2}+x_{2}^{2}}{4}$, $C_{1}, C_{2}$-are arbitrary constants, are invariant solutions of Equation (37), with respect to the group of transformations generated by the vector field (38). Here BesselJ, BesselY are the Bessel functions of the first kind and the second kind, respectively.

Proof. The flow of the vector field $X$ consists of transformations

$$
\left(t, x_{1}, x_{2}, u\right) \rightarrow\left(t \mathrm{e}^{2 s}, x_{1}, x_{2}, u-2 s\right), s \in \mathbb{R}
$$

Using the above criterion we find that the function

$$
I=\frac{\exp (u / 2) \sqrt{t}}{V(\xi)}
$$

where $\xi=\xi\left(x_{1}, x_{2}\right)$, is an invariant function of the transformation group (40), which follows from the following equalities $X(I)=0$.

The invariant function allows us to find a solution in the form

$$
u\left(t, x_{1}, x_{2}\right)=\ln \frac{V(\xi)}{t}, \xi=\frac{x_{1}^{2}+x_{2}^{2}}{4} \text {. }
$$

Then for the function $V(\xi)$ we get the following second-order differential equation

$$
\xi V^{\prime \prime}+V^{\prime}+V+1=0
$$

The general solution of Equation (42) is given by the following functions.

$$
V(\xi)=C_{1} \operatorname{BesselJ}(0,2 \sqrt{\xi})+C_{2} \operatorname{Bessel} Y(0,2 \sqrt{\xi})+1,
$$

$\xi=\frac{x_{1}^{2}+x_{2}^{2}}{4}, C_{1}, C_{2}$-are arbitrary constants.

\section{Conclusion}

Summarizing the results we can conclude that using infinitesimal generators of symmetry groups to find solutions of second-order partial differential equations, we obtain the ordinary differential equation of the second order. If this ordinary differential equation of the second integrates explicitly, we get large class invariant solutions of a given partial differential equation. Among these solutions there can be exact solutions that can be used to characterize the process that is described by this equation. It all depends on the choice of invariant functions. Often, the obtained second-order ordinary differential equation is explicitly not integrated. In this case, we cannot find invariant solutions in explicit form, but we can characterize the behavior of solutions using numerical integration. 


\section{Conflicts of Interest}

The author declares no conflicts of interest regarding the publication of this paper.

\section{References}

[1] Lie, S. and Sheffers, G. (2011) Symmetries of Differential Equations. Vol. 1. Lectures on Differential Equations with Known Infinitesimal Transformations. In: Regular and Chaotic Dynamics, Izhevsk, Moscow, 704 p.

[2] Lie, S. and Sheffers, G. (2013) Symmetries of Differential Equations. Vol. 3. In: Geometry of Contact Transformations, Udmurtskiy Gosudarstvenniy Universitet, Izhevsk, 702.

[3] Ayub, M., Khan, M. and Mahomed, F.M. (2013) Second-Order Systems of ODEs Admitting Three-Dimensional Lie Algebras and Integrability. Journal of Applied Mathematics, 2013, Article ID: 147921. https://doi.org/10.1155/2013/147921

[4] Wafo, S.C. and Mahomed, F.M. (2004) Reduction of Order for Systems of Ordinary Differential Equations. Journal of Nonlinear Mathematical Physics, 11, 13-20. https://doi.org/10.2991/jnmp.2004.11.1.3

[5] Olver, P.J. (1986) Applications of Lie Groups to Differential Equations. Springer, Berlin, 513 p. https://doi.org/10.1007/978-1-4684-0274-2

[6] Ovsiannikov, L.V. (1982) Group Analysis of Differential Equations. Academic Press, Cambridge, 432 p. https://doi.org/10.1016/C2013-0-07470-1

[7] Dorodnitsyn, V.A., Knyazeva, I.V. and Svirshchevskii, S.R. (1983) Group Properties of the Heat Equation with Source in the Two-Dimensional and Three-Dimensional Cases. Differential Equations, 19, 1215-1223.

[8] Narmanov, O.A. (2018) Lie Algebra of Infinitesimal Generators of the Symmetry Group of the Heat Equation. Journal of Applied Mathematics and Physics, 6, 373-381. https://doi.org/10.4236/jamp.2018.62035

[9] Samarskiy, A.A., Galaktionov, V.A., Kurdumov, S.P. and Mikhaylov, A.P. (1987) Rejimy s obostreniyem $\mathrm{v}$ zadachax dlya kvazilineynyx parabolicheskix uravneniy. Nauka, Moscow, 481 p. (In Russian) 